\title{
Elevated Levels of High Sensitivity C-Reactive Protein are Associated with Metabolic Syndrome in a Representative Korean Sample
}

\author{
Hye-Sun Shin, PhD* \\ Department of Dental Hygiene, Eulji University College of Health Science, Seongnam, South Korea \\ *Corresponding Author: Hye-Sun Shin, Department of Dental Hygiene, Eulji University College of Health \\ Science, 553 Sanseong-daero, Sujeong-gu, Seongnam 13135, South Korea, Email: bbanna82@snu.ac.kr
}

\begin{abstract}
Background: To date, levels of high-sensitivity C-reactive protein (hsCRP) have not been reported in the Korean national data. The purpose of this study was to investigate whether hsCRP levels are associated with metabolic syndrome (MetS) in a representative sample of the Korean population.
\end{abstract}

Methods: A cross-sectional study was conducted on 4,546 participants > 19 years of age using the 2015 Korea National Health and Nutrition Examination Survey data. hsCRP concentrations were divided into quartiles. MetS was defined using the criteria of the Joint Interim Statement of the International Diabetes Federation. Confounding variables were demographic factors, socioeconomic status, general health behaviors, and systemic health status.

Results: Significant differences in MetS and its components were observed according to the hsCRP quartiles $(P<0.001)$. The hsCRP quartiles were significantly associated with MetS and its components in the fully adjusted model with a dose-response relationship (3rd quartile: odds ratio [OR] $=1.95$; 95\% confidence interval $[C I]=1.48-2.56$ and 4th quartile: $O R=2.58,95 \% C I=1.91-3.49$ for MetS). hsCRP level increased as the number of MetS components present increased. In women, the fully adjusted OR for MetS was 2.25 (95\% CI $=1.58-3.19)$ for the 3rd quartile and 3.77 (95\% CI $=2.50-5.69)$ for the 4th quartile.

Conclusions: Our findings suggest that an increase in hsCRP level may be independently associated with MetS in Korean adults.

Keywords: Epidemiology; KNHANES; high sensitivity C reactive protein; metabolic syndrome

\section{INTRODUCTION}

Metabolic syndrome (MetS) defines a group of cardiovascular risk factors affecting the development of cardiovascular disease and type 2 diabetes [1,2]. The most common abnorm alities of MetS components are abdominal obesity, elevated triglycerides (TG), reduced high-density lipoprotein (HDL), elevated blood pressure, and elevated fasting glucose [3]. The prevalence of MetS in the 2011-2012 Korea National Health and Nutrition Examination Survey (KNHANES) data was 31.3\% [4].

High sensitivity C-reactive protein (hsCRP) is a crucial marker of systemic low grade inflammation [5,6]. Epidemiological studies have shown a strong positive association between elevated levels of hsCRP and MetS and its components $[7,8,9,10]$. It has also been proposed that hsCRP be added as a clinical criterion for MetS [11].

Several studies have evaluated the association between hsCRP and MetS [7,11,12,13,14]. A significant association has been reported between hsCRP and MetS in the United States using data from the National Health and Nutrition Examination Survey [8,9]. In addition, a few studies have investigated the association between hsCRP and MetS in the Asian population [15-17]. However, previous Asian population studies are limited to hospital-based patient [15], community-dwelling women [17], and health screening populations $[10,16]$, which makes it difficult to generalize the results. No study has investigated the distribution of hsCRP concentrations in a representative sample of the Korean population. Thus, we analyzed hsCRP concentration for the first time in the 2015 KNHANES data. 
We hypothesized that elevated hsCRP level is associated with MetS and its components in a representative sample of the Korean population.

The objectives of this study were 1) to analyze concentrations of hsCRP as a new inflammatory marker in a representative sample of the Korean population; 2) to compare hsCRP levels with previous reports, and 3) to examine the association between elevated levels of hsCRP and MetS after considering possible confounders.

\section{Materials AND Methods}

\subsection{Study Design and Participants}

Data were extracted from the 2015 KNHANES, which is a study periodically conducted by the Korea Center for Disease Control and Prevention. The sampling protocol was designed to include a complex, stratified, multistage, and probability-cluster survey of a representative sample of the non-institutionalized civilian population. The participants were randomly selected by geographic area, age, and gender based on the 2005 National Census Registry. The survey consisted of a health interview, a nutrition survey, and a health and oral examination survey. All participants provided written informed consent before participating in the survey. The total number of participants in the 2015 KNHANES was 7,380 (3,381 men and 3,999 women).

Inclusion criteria were: 1) $\geq 19$ years of age; 2) laboratory tested for MetS; 3) blood hsCRP level measured; and 4) no missing values for the potential confounders. The final sample comprised 4,546 participants (1,990 men and 2,556 women).

\subsection{Measurement of Hscrp}

Blood was collected, stored at $2^{\circ} \sim 8^{\circ} \mathrm{C}$, and transferred to the central laboratory (NeoDin Medical Institute, Seoul, Korea) within 24 hours for the hsCRP analysis. hsCRP concentrations were measured by immunoturbidimetry using the Cobas instrument (Roche, Mannheim, Germany). The limit of detection was $0.09 \mathrm{mg} / \mathrm{L}$ and the maximum value measurable in the assay was $20.01 \mathrm{mg} / \mathrm{L}$. The hsCRP concentrations were divided into quartiles for statistical analysis, as follows: 1st quartile (lowest): 0.09$0.3 \mathrm{mg} / \mathrm{L}$; 2nd quartile: $0.4-0.5 \mathrm{mg} / \mathrm{L}$; 3rd quartile: $0.6-1.0 \mathrm{mg} / \mathrm{L}$; and 4 th quartile (highest): $1.1-20.01 \mathrm{mg} / \mathrm{L}$ ).

\subsection{Assessment of Mets}

MetS was defined using the criteria of the Joint Interim Statement of the International Diabetes Federation Task Force on Epidemiology and Prevention [18]. The MetS assessment consisted of the following five metabolic components: waist circumference in the Asian population [19] ( $\geq 90 \mathrm{~cm}$ for men and $\geq 80 \mathrm{~cm}$ for women); elevated TG $(\geq 150 \mathrm{mg} / \mathrm{dL}$ or taking medication for hypertriglyceridemia); low HDL-cholesterol ( $<40 \mathrm{mg} / \mathrm{dL}$ in men and $<50 \mathrm{mg} / \mathrm{dL}$ in women); high blood pressure (systolic blood pressure $\geq$ $130 \mathrm{~mm} \mathrm{Hg}$ or diastolic blood pressure $\geq 85 \mathrm{~mm}$ $\mathrm{Hg}$ or taking medication for hypertension); and high fasting glucose $(\geq 100 \mathrm{mg} / \mathrm{dL})$. MetS was defined as the presence of at least three of these components.

\subsection{Assessment of Confounders}

Data on confounding variables employed in the statistical analysis considered information on sociodemographic factors (age, gender, income, and education), general health behaviors (smoking, drinking, and physical activity), and systemic health status (diabetes mellitus and obesity).

Household income was the monthly average family income and was divided into quartiles. Education level was divided into four groups of less than primary school, middle school, high school, and college or higher. The participants were asked whether they were currently smoking and the answers were categorized into: no (never smoker and past smoker) and yes (current smoker). Alcohol consumption was categorized into: non-drinker, almost nondrinker ( $\leq 1$ day per month), light drinker (2-4 days per month), moderate drinker ( $2-3$ days per week), and heavy drinker ( $\geq 4$ days per week). Drinking was dichotomized into: no (none/almost non-drinker) and yes (light/ moderate/heavy drinker). Physical activity was assessed by aerobic physical activity as follows: $\geq 150 \mathrm{~min}$ per week of moderate-intensity aerobic physical activity, or $75 \mathrm{~min}$ per week of vigorous-intensity aerobic physical activity, or an equivalent combination of moderate-and vigorous-intensity aerobic physical activity [20]. Diabetes mellitus was defined as having a fasting glucose level > $126 \mathrm{mg} / \mathrm{dL}$ or taking medication for diabetes. Body mass index (BMI) was used to define obesity. BMI was calculated by dividing weight in kilograms by the square of height in meters. Obesity was defined as a $B M I \geq 25.0 \mathrm{~kg} / \mathrm{m}^{2}$. 


\subsection{Statistical Analysis}

The dependent variable was MetS and the independent variable was hsCRP concentration. Differences in the general characteristics of the participants were compared according to the presence of MetS using the chi-square test. We presented the distribution of MetS and its components according to hsCRP quartiles using the chi-square test. All data are presented as weighted percentages and standard errors (SEs). The distributions of the quartiles of hsCRP and the MetS components as continuous confounding variables were expressed as the unadjusted and adjusted mean and SE using analysis of variance and analysis of covariance. A multivariable logistic regression analysis was performed to examine the associations between hsCRP level and MetS and its components. A multivariable logistic regression was used to compute unadjusted and adjusted odds ratios (ORs) and confidence intervals (CIs). Model 1 was unadjusted. Model 2 was adjusted for demographic variables, including age and gender. Model 3 was adjusted for demographic factors, socioeconomic status, and general health variables, including income, education, smoking, drinking, and physical activity. Model 4 was adjusted for all confounding variables, including diabetes mellitus and obesity. We also evaluated the associations between hsCRP level and number of MetS components by classifying the participants according to the total number of MetS components. We performed a subgroup analysis by gender. All statistical analyses were performed using the SPSS 19.0 (SPSS Inc., Chicago, IL, USA) statistical program. A $P$ value $<0.05$ was considered significant.

\section{RESULTS}

\subsection{Characteristics of the Participants}

Of the 4,546 participants, the prevalence of MetS was estimated to be $32.6 \%$. Table 1 shows the characteristics of the study participants according to the results of the MetS diagnosis. The data indicate that most patients with MetS were $\geq 40$ years compared to those without Mets, and there were associations with male gender, lower income, primary school, current smoker, non-drinker, and no physical activity. The percentage of participants with MetS was significantly higher in the diabetes mellitus and obesity. The mean hsCRP level in the MetS group was significantly higher than that in the group without MetS (no MetS: $0.64 \mathrm{mg} / \mathrm{L}$; MetS: $1.61 \mathrm{mg} / \mathrm{L}$, respectively). Participants with MetS were significantly more likely to have a high hsCRP level than those without.

\subsection{Distribution of Mets and its Components According to Hscrp Quartiles}

Significant differences in MetS and its components were observed according to the hsCRP quartiles $(P<0.001$ for all). Elevated hsCRP levelswere distributed in participants with abdominal obesity, high TG, low HDL, high blood pressure, and high fasting glucose (Table 2).

\subsection{Association between hsCRP Quartiles and MetS and its Components}

The hsCRP quartiles were consistently associated with MetS and all components in the logistic models throughout the adjustment process (Table 3). In crude analyses, the OR of MetS in the highest hsCRP quartile was 5.56 compared to participants in the lowest hsCRP quartile. After adjusting for demographic variables, socioeconomic status, and general health behaviors in that order, the association between hsCRP quartiles and MetS was slightly weakened. Especially, after including systemic health status, the association between the second hsCRP quartile and MetS disappeared (3rd quartile: $\mathrm{OR}=1.95,95 \% \mathrm{CI}=1.48-2.56$ and 4th quartile: $\mathrm{OR}=2.58,95 \% \mathrm{CI}=1.91-3.49$ ). The adjusted ORs of the highest hsCRP quartile for each MetS component were as follows: 2.53 for abdominal obesity; 2.03 for high TGs; 2.53 for low HDL cholesterol; 1.31 for high blood pressure; and 1.75 for high fasting glucose.

\subsection{Association between hsCRP Quartiles and MetS Components}

The hsCRP quartiles also showed a doseresponse relationship with increases in the number of MetS components (3rd quartile: OR $=1.55$ and 4th quartile: $\mathrm{OR}=1.76$ for three MetS components; 3rd quartile: $\mathrm{OR}=2.16$ and 4 th quartile $=\mathrm{OR}=2.41$ for four MetS components; 3rd quartile: $\mathrm{OR}=2.51$ and 4 th quartile: $\mathrm{OR}=2.72$ for five MetS components). The strength of the association was highest in the five MetS components group (Table 4).

\subsection{Gender Stratified Associations between Hscrp Quartiles and Mets}

The association between hsCRP quartiles and MetS was stronger in women than that in the total population (Table 5). The fully adjusted OR was 2.25 (95\% CI: 1.58-3.19) for the 3rd quartile and 3.77 (95\% CI: $2.50-5.69)$ for the 4 th quartile. 
Elevated Levels of High Sensitivity C-Reactive Protein are Associated with Metabolic Syndrome in a Representative Korean Sample

Table1. Characteristics of the participants $(N=4,546)$

\begin{tabular}{|c|c|c|c|c|}
\hline & & \multicolumn{2}{|c|}{ Metabolic syndrome } & \multirow[b]{3}{*}{$P$ value ${ }^{*}$} \\
\hline & & Non-MetS & MetS & \\
\hline Variables & Total n & $(n=3,063)$ & $(n=1,483)$ & \\
\hline \multicolumn{5}{|l|}{ Age (years) } \\
\hline $20-39$ & 1149 & $44.4(1.3)$ & $14.4(1.3)$ & $<0.001$ \\
\hline $40-59$ & 1768 & $39.3(1.2)$ & $45.5(1.7)$ & \\
\hline$\geq 60$ & 1629 & $16.2(0.8)$ & $40.0(1.6)$ & \\
\hline \multicolumn{5}{|l|}{ Gender } \\
\hline Men & 1990 & $48.3(1.0)$ & $53.0(1.3)$ & 0.010 \\
\hline Women & 2556 & $51.7(1.0)$ & $47.0(1.3)$ & \\
\hline \multicolumn{5}{|l|}{ Monthly Household income } \\
\hline Lowest quartile & 1051 & $22.0(1.3)$ & $26.5(1.5)$ & 0.047 \\
\hline Lower middle quartile & 1139 & $25.2(1.3)$ & $25.0(1.6)$ & \\
\hline Upper middle quartile & 1187 & $26.3(1.2)$ & $24.7(1.4)$ & \\
\hline Highest quartile & 1169 & $26.5(1.6)$ & $23.8(1.5)$ & \\
\hline \multicolumn{5}{|l|}{ Education } \\
\hline Primary school & 981 & $9.3(0.6)$ & $29.2(1.6)$ & $<0.001$ \\
\hline Middle school & 496 & $7.5(0.6)$ & $11.7(1.0)$ & \\
\hline High school & 1567 & $40.6(1.3)$ & $31.1(1.6)$ & \\
\hline College & 1502 & $42.7(1.5)$ & $27.9(1.6)$ & \\
\hline \multicolumn{5}{|l|}{ Smoking } \\
\hline No & 3783 & $80.6(0.9)$ & $76.5(1.5)$ & 0.012 \\
\hline Yes & 763 & $19.4(0.9)$ & $23.5(1.5)$ & \\
\hline \multicolumn{5}{|l|}{ Drinking } \\
\hline No & 1241 & $20.0(0.9)$ & $29.5(1.3)$ & $<0.001$ \\
\hline Yes & 3305 & $80.0(0.9)$ & $70.5(1.3)$ & \\
\hline \multicolumn{5}{|l|}{ Physical activity } \\
\hline No & 2377 & $45.1(1.2)$ & $56.0(1.8)$ & $<0.001$ \\
\hline Yes & 2169 & $54.9(1.2)$ & $44.0(1.8)$ & \\
\hline \multicolumn{5}{|l|}{ Diabetes mellitus } \\
\hline No & 4025 & $96.8(0.3)$ & $77.3(1.2)$ & $<0.001$ \\
\hline Yes & 521 & $3.2(0.3)$ & $22.7(1.2)$ & \\
\hline \multicolumn{5}{|l|}{ Obesity } \\
\hline No & 2992 & $79.5(0.9)$ & $33.1(1.5)$ & $<0.001$ \\
\hline Yes & 1554 & $20.5(0.9)$ & $66.9(1.5)$ & \\
\hline hsCRP (mg/L) & 4546 & $0.64(0.05)$ & $1.61(0.07)$ & $<0.001^{\dagger}$ \\
\hline \multicolumn{5}{|l|}{ hsCRP (quartiles) } \\
\hline I $\quad(0.09-0.3 \mathrm{mg} / \mathrm{L})($ Lowest $)$ & 1140 & $31.4(1.4)$ & $11.8(1.2)$ & $<0.001$ \\
\hline II $(0.4-0.5 \mathrm{mg} / \mathrm{L})$ & 1043 & $26.5(1.1)$ & $17.8(1.3)$ & \\
\hline III $\quad(0.6-1.0 \mathrm{mg} / \mathrm{L})$ & 1183 & $23.2(0.9)$ & $30.8(1.5)$ & \\
\hline IV $\quad(1.1-20.01 \mathrm{mg} / \mathrm{L})($ Highest $)$ & 1180 & $19.0(0.9)$ & $39.7(1.6)$ & \\
\hline
\end{tabular}

Data were presented as weighted percentage and standard error.

* Obtained from chi-square test.

Obtained from independent $t$-test.

Bold denotes statistical significance at $P<0.05$.

Table2. Distribuion of metabolic syndrome and its components according to hs CRP quartiles

\begin{tabular}{|l|c|c|c|c|c|c|}
\hline & & \multicolumn{2}{|c|}{ Quartile of hsCRP level } & & \\
\hline & & I (lowest) & II & III & IV(highest) & \\
\hline MetS components & $\mathrm{N}$ & $(\mathrm{n}=1,140)$ & $(\mathrm{n}=1,043)$ & $(\mathrm{n}=1,183)$ & $(\mathrm{n}=1,180)$ & $P$ value $^{*}$ \\
\hline MetS syndrome & & & & & & \\
\hline No & 3063 & $87.3(1.2)$ & $79.5(1.5)$ & $66.1(1.7)$ & $55.4(1.8)$ & $<\mathbf{0 . 0 0 1}$ \\
\hline Yes & 1483 & $12.7(1.2)$ & $20.5(1.5)$ & $33.9(1.7)$ & $44.6(1.8)$ & \\
\hline Waist circumference & & & & & & \\
\hline$<90(80)$ & 2697 & $80.8(1.5)$ & $70.6(1.7)$ & $55.6(1.6)$ & $45.4(1.8)$ & $<\mathbf{0 . 0 0 1}$ \\
\hline$\geq 90(80)$ & 1849 & $19.2(1.5)$ & $29.4(1.7)$ & $44.4(1.6)$ & $54.6(1.8)$ & \\
\hline
\end{tabular}


Elevated Levels of High Sensitivity C-Reactive Protein are Associated with Metabolic Syndrome in a Representative Korean Sample

\begin{tabular}{|c|c|c|c|c|c|c|}
\hline \multicolumn{2}{|c|}{ Triglycerides } & & & & & \\
\hline$<150$ & 3251 & $85.1(1.3)$ & $75.5(1.6)$ & $65.6(1.8)$ & $61.4(1.7)$ & $<0.001$ \\
\hline$\geq 150$ & 1295 & $14.9(1.3)$ & $24.5(1.6)$ & $34.4(1.8)$ & $38.6(1.7)$ & \\
\hline \multicolumn{2}{|c|}{ HDL cholesterol } & & & & & \\
\hline$\geq 40(50)$ & 2948 & $79.6(1.4)$ & $75.7(1.5)$ & $64.3(1.6)$ & $53.9(1.9)$ & $<0.001$ \\
\hline$<40(50)$ & 1598 & $20.4(1.4)$ & $24.3(1.5)$ & $35.7(1.6)$ & $46.1(1.9)$ & \\
\hline \multicolumn{2}{|c|}{ Blood pressure } & & & & & \\
\hline No & 2671 & $77.9(1.5)$ & $69.0(1.9)$ & $59.8(1.9)$ & $56.8(1.9)$ & $<0.001$ \\
\hline Yes & 1875 & $22.1(1.5)$ & $31.0(1.9)$ & $40.2(1.9)$ & $43.2(1.9)$ & \\
\hline \multicolumn{2}{|c|}{ Fasting blood glucose } & & & & & \\
\hline$<100$ & 2923 & $80.1(1.4)$ & $72.2(1.5)$ & $65.2(1.8)$ & $56.6(1.8)$ & $<0.001$ \\
\hline$\geq 100$ & 1623 & $19.9(1.4)$ & $27.8(1.5)$ & $34.8(1.8)$ & $43.4(1.8)$ & \\
\hline
\end{tabular}

Data were presented as weighted percentage and standard error.

* Obtained from chi-square test.

${ }^{*}$ Metabolic syndrome was defined as at least three of the metabolic components.

Bold denotes statistical significance at $P<0.05$

Table3. Association between hsCRP quartiles and metabolic syndrome and its components

\begin{tabular}{|c|c|c|c|c|}
\hline & \multicolumn{4}{|c|}{ Odds ratio ( $95 \%$ confidence interval) } \\
\hline & Model 1 & Model 2 & Model 3 & Model 4 \\
\hline \multicolumn{5}{|l|}{$\operatorname{MetS}(\geq 3)$} \\
\hline I (lowest) & Reference & Reference & Reference & Reference \\
\hline II & $1.78(1.34-2.37)$ & $1.59(1.19-2.1)$ & $1.57(1.18-2.09)$ & $1.34(0.97-1.84)$ \\
\hline III & $3.54(2.76-4.52)$ & $2.93(2.30-3.75)$ & $2.77(2.16-3.55)$ & $1.95(1.48-2.56)$ \\
\hline IV (highest) & $5.56(4.26-7.27)$ & $5.04(3.85-6.60)$ & $4.59(3.50-6.03)$ & $2.58(1.91-3.49)$ \\
\hline \multicolumn{5}{|c|}{ Abdominal obesity } \\
\hline I (lowest) & Reference & Reference & Reference & Reference \\
\hline II & $1.75(1.36-2.25)$ & $1.80(1.40-2.32)$ & $1.79(1.39-2.31)$ & $1.53(1.14-2.05)$ \\
\hline III & $3.36(2.66-4.26)$ & $3.35(2.64-4.27)$ & $3.28(2.57-4.18)$ & $2.23(1.64-3.03)$ \\
\hline IV (highest) & $5.06(3.94-6.51)$ & $5.28(4.06-6.87)$ & $5.02(3.87-6.51)$ & $2.53(1.89-3.39)$ \\
\hline \multicolumn{5}{|c|}{ High triglycerides } \\
\hline I (lowest) & Reference & Reference & Reference & Reference \\
\hline II & $1.85(1.39-2.46)$ & $1.59(1.19-2.18)$ & $1.58(1.18-2.11)$ & $1.44(1.07-1.94)$ \\
\hline III & $3.00(2.32-3.88)$ & $2.50(1.93-3.25)$ & $2.41(1.86-3.14)$ & $1.96(1.50-2.58)$ \\
\hline IV (highest) & $3.59(2.81-4.59)$ & $3.09(2.39-3.99)$ & $2.87(2.21-3.74)$ & $2.03(1.53-2.68)$ \\
\hline \multicolumn{5}{|c|}{ Low HDL cholesterol } \\
\hline I (lowest) & Reference & Reference & Reference & Reference \\
\hline II & $1.25(0.98-1.60)$ & $1.30(1.02-1.66)$ & $1.29(1.01-1.65)$ & $1.21(0.95-1.54)$ \\
\hline III & $2.17(1.74-2.70)$ & $2.20(1.77-2.74)$ & $2.13(1.71-2.66)$ & $1.82(1.47-2.27)$ \\
\hline IV (highest) & $3.33(2.69-4.13)$ & $3.52(2.85-4.35)$ & $3.29(2.67-4.05)$ & $2.53(2.06-3.12)$ \\
\hline \multicolumn{5}{|c|}{ High blood pressure } \\
\hline I (lowest) & Reference & Reference & Reference & Reference \\
\hline II & $1.58(1.25-2.00)$ & $1.32(1.02-1.71)$ & $1.30(1.00-1.67)$ & $1.17(0.90-1.52)$ \\
\hline III & $2.36(1.91-2.93)$ & $1.75(1.39-2.20)$ & $1.63(1.29-2.06)$ & $1.30(1.04-1.64)$ \\
\hline IV (highest) & $2.67(2.15-3.32)$ & $2.09(1.67-2.63)$ & $1.88(1.49-2.35)$ & $1.31(1.04-1.64)$ \\
\hline \multicolumn{5}{|c|}{ High fasting glucose } \\
\hline I (lowest) & Reference & Reference & Reference & Reference \\
\hline II & $1.55(1.25-1.92)$ & $1.30(1.03-1.63)$ & $1.29(1.03-1.61)$ & $1.23(0.95-1.58)$ \\
\hline III & $2.14(1.73-2.66)$ & $1.61(1.29-2.03)$ & $1.57(1.25-1.96)$ & $1.29(1.01-1.64)$ \\
\hline IV (highest) & $3.08(2.47-3.83)$ & $2.51(1.98-3.19)$ & $2.41(1.89-3.07)$ & $1.75(1.33-2.30)$ \\
\hline
\end{tabular}

Dependent variables: Metabolic syndrome and its components

Model 1 was unadjusted association.

Model 2 was adjusted for age and gender

Model 3 was adjusted for age, gender, income, education, smoking, drinking, and physical activity

Model 4 was adjusted for age, gender, income, education, smoking, drinking, physical activity, diabetes mellitus, and obesity

Bold denotes statistical significance at $P<0.05$

ARC Journal of Public Health and Community Medicine 
Elevated Levels of High Sensitivity C-Reactive Protein are Associated with Metabolic Syndrome in a Representative Korean Sample

Table4. Association between hSCRP quartiles and the number of MetS components

\begin{tabular}{|c|c|c|c|c|}
\hline & \multicolumn{4}{|c|}{ Odds ratio ( $95 \%$ confidence interval) } \\
\hline & Model 1 & Model 2 & Model 3 & Model 4 \\
\hline \multicolumn{5}{|c|}{$\operatorname{MetS}(3)(n=816)$} \\
\hline I (lowest) & Reference & Reference & Reference & Reference \\
\hline II & $1.53(1.11-2.12)$ & $1.37(1.00-1.88)$ & $1.35(0.98-1.86)$ & $1.20(0.87-1.66)$ \\
\hline III & $2.48(1.89-3.26)$ & $2.05(1.55-2.70)$ & $1.98(1.50-2.63)$ & $1.55(1.16-2.06)$ \\
\hline IV (highest) & $3.21(2.40-4.27)$ & $2.73(2.04-3.67)$ & $2.61(1.94-3.53)$ & $1.76(1.28-2.41)$ \\
\hline \multicolumn{5}{|c|}{$\operatorname{MetS}(4) \quad(n=496)$} \\
\hline I (lowest) & Reference & Reference & Reference & Reference \\
\hline II & $1.80(1.15-2.81)$ & $1.64(1.05-2.56)$ & $1.62(1.04-2.54)$ & $1.42(0.91-2.21)$ \\
\hline III & $3.81(2.67-5.43)$ & $3.17(2.23-4.50)$ & $3.00(2.10-4.27)$ & $2.16(1.50-3.11)$ \\
\hline IV (highest) & $5.58(3.82-8.15)$ & $4.79(3.26-7.06)$ & $4.32(2.95-6.34)$ & $2.41(1.64-3.53)$ \\
\hline \multicolumn{5}{|c|}{$\operatorname{MetS}(5) \quad(n=171)$} \\
\hline I (lowest) & Reference & Reference & Reference & Reference \\
\hline II & $2.74(1.18-6.35)$ & $2.44(1.05-5.64)$ & $2.41(1.05-5.53)$ & $2.14(0.98-4.68)$ \\
\hline III & $4.56(2.22-9.37)$ & $3.63(1.75-7.53)$ & $3.39(1.65-6.96)$ & $2.51(1.23-5.14)$ \\
\hline IV (highest) & $7.32(3.50-15.30)$ & $5.99(2.85-12.62)$ & $5.35(2.54-11.24)$ & $2.72(1.31-5.62)$ \\
\hline
\end{tabular}

Dependent variable: Metabolic syndrome

Model 1 was unadjusted association.

Model 2 was adjusted for age and gender

Model 3 was adjusted for age, gender, income, education, smoking, drinking, and physical activity

Model 4 was adjusted for age, gender, income, education, smoking, drinking, physical activity, diabetes mellitus, and obesity

Bold denotes statistical significance at $P<0.05$

Table5. Gender stratified-association between hsCRP quartiles and metabolic syndrome

\begin{tabular}{|l|c|c|c|}
\hline & \multicolumn{3}{|c|}{ Odds ratio $(\mathbf{9 5 \%}$ confidence interval) } \\
\hline & Total $(\mathbf{n = 4 , 5 4 6})$ & Men $(\mathbf{n = 1 , 9 9 0})$ & Women $(\mathbf{n = 2 , 5 5 6 )}$ \\
\hline MetS $(\geq 3)$ & & & Reference \\
\hline I (lowest) & Reference & Reference & $1.39(0.89-2.16)$ \\
\hline II & $1.34(0.97-1.84)$ & $1.11(0.71-1.74)$ & $2.25(1.58-3.19)$ \\
\hline III & $1.95(1.48-2.56)$ & $1.55(1.06-2.27)$ & $3.77(2.50-5.69)$ \\
\hline IV (highest) & $2.58(1.91-3.49)$ & $1.99(1.29-3.05)$ & \\
\hline
\end{tabular}

Dependent variables: Metabolic syndrome

Model was adjusted for age, gender, income, education, smoking, drinking, physical activity, diabetes mellitus, and obesity.

Bold denotes statistical significance at $P<0.05$

\section{DisCUSSION}

Increased hsCRP level was associated with increased odds for MetS and its components after adjusting for age, gender, income, education, smoking, drinking, physical activity, diabetes mellitus, and obesity. To the best of our knowledge, this is the first study to report an association between hsCRP and MetS in a representative sample of the Korean population. Moreover, we evaluated various confounders, such as socioeconomic status, general health behaviors, and systemic health status that could affect MetS.

Although the analysis of retrospective study data from 13,426 participants showed an association between the highest hsCRP quartile and MetS, the same results for each MetS component were not present in Koreans [10]. Because the study participants were mainly healthy adults, which limits generalizability, the prevalence of MetS was very low, unlike the prevalence of MetS in the general population. In a Japanese study of community-dwelling women, the ORs of hsCRP were significantly higher for MetS $(\mathrm{OR}=3.23)$ and its components after controlling for age, smoking, and alcohol consumption [17]. Our results showed a stronger association after adjusting for demographics, socioeconomic status, and general health variables than that in the previous study (OR = 4.59). We considered diabetes mellitus and obesity as major risk factors for MetS and the strength of the association with MetS and its 
components was considerably weakened. In a cross-sectional study, urban Indians with elevated hsCRP levels had a weaker association with MetS compared with the present results [15]. Thus, the results are difficult to compare directly because the study populations differ, different MetS criteria and confounders were used, and the hsCRP quartiles differed in previous studies.

Although hsCRP concentrations differ in different populations, it is unclear why they are elevated in specific groups. Our results show that mean hsCRP levels in men and women were 1.37 and $1.18 \mathrm{mg} / \mathrm{L}$, respectively. Gender discrepancies in hsCRP have been observed consistently [21, 22]. Women in our population had the lowest mean hsCRP level compared to other ethnicities, followed by African-American $(5.85 \mathrm{mg} / \mathrm{L})$, Hispanic $(3.90 \mathrm{mg} / \mathrm{L})$, Caucasian (3.45 mg/L), Chinese (1.44 mg/L), and Japanese participants $(1.37 \mathrm{mg} / \mathrm{L})$ [23].

The American Heart Association (AHA) and the US Centers for Disease Control and Prevention (CDC) suggest three hsCRP cut-off categories for stratifying the risk of cardiovascular disease events: < $1 \mathrm{mg} / \mathrm{L}$ (low risk), $1-3 \mathrm{mg} / \mathrm{L}$ (average risk), and $>3 \mathrm{mg} / \mathrm{L}$ (high risk) [24]. The risk assessment tool has limitations for applying to other ethnic populations [24] and this point needs to be addressed in a further Asian population study. Based on our findings, the ORs of MetS were similar in average risk and high risk according to the AHA/CDC categories, which is inconsistent with previous studies [17, 25]. When considering the results of the hsCRP quartiles, the risk for MetS and its components in the higher hsCRP quartile was significantly higher than that in the lower hsCRP quartile. We speculate that even Korean adults with low hsCRP concentrations may be susceptible to the development of MetS.

The hsCRP quartiles also showed a doseresponse relationship with increases in the MetS components, similar to the results of previous studies [7, 14]. In agreement with previous studies [15, 16], our findings showed remarkable differences by gender in the association between hsCRP and MetS. This observation may have been affected by age- and gender-related factors [26]. The prevalence of MetS increased with age in a gender-specific manner. The prevalence of MetS is slightly higher in men $<50$ years of age, but it reverses after 50 years of age [26]. Gender differences related to genetic and biological pathways are reportedly caused by hyperandrogenism, insulin-resistance, and the associated increase in abdominal obesity and reduced HDL-cholesterol that occur after menopause [26]. We speculate that women are also more prone to develop MetS than men.

hsCRP quartiles were moderately associated with individual MetS components. In particular, several studies have reported that abdominal obesity is the primary determinant of elevated hsCRP levels in patients with Mets components [27,28,29]. Significant associations with MetS were found for abdominal obesity and low HDL-cholesterol rather than the other components in our study. These results regarding low HDL-cholesterol remain controversial $[15,16]$, so further research is needed.

Nevertheless, this cross-sectional study cannot infer causal relationships. Further well-designed longitudinal studies are required to identify the present associations. However, our study had several major strengths. Anthropometric assessments and blood analyses were performed clinically by physicians, trained examiners, and trained researchers. Although there is some evidence for an association between hsCRP and MetS [15-17] in Asian populations, no study has been conducted on a representative sample of Koreans. Thus, we analyzed newly released data on hsCRP from KNHANES 2015 and identified the distribution of hsCRP concentration in the Korean population. These results provide the first evidence for an association between hsCRP and MetS and its components in a representative sample of Korean adults. Well-designed longitudinal studies will be necessary to clarify the effect of hsCRP on MetS and to establish the cutoff points for elevated hsCRP as a clinical assessment tool for MetS among Korean adults.

\section{REFERENCES}

[1] Sattar N, McConnachie A, Shaper AG, Blauw GJ, Buckley BM, et al. Can metabolic syndrome usefully predict cardiovascular disease and diabetes? Outcome data from two prospective studies. Lancet 2008;371:19271935.

[2] Rutter MK, Meigs JB, Sullivan LM, D'Agostino RB, Sr., Wilson PW. C-reactive protein, the metabolic syndrome, and prediction of cardiovascular events in the Framingham Offspring Study. Circulation 2004;110:380-385.

[3] Eckel RH, Grundy SM, Zimmet PZ. The metabolic syndrome. Lancet 2005;365:14151428. 
[4] Park SY, Park YK, Cho KH, Choi HJ, Han JH, et al. Normal range albuminuria and metabolic syndrome in South Korea: the 2011-2012 Korean National Health and Nutrition Examination Survey. PloS one 2015;10:e0125615.

[5] Ridker PM, Hennekens CH, Buring JE, Rifai N. C-reactive protein and other markers of inflammation in the prediction of cardiovascular disease in women. N Engl J Med 2000;342:836-843.

[6] Freeman DJ, Norrie J, Caslake MJ, Gaw A, Ford I, et al. C-reactive protein is an independent predictor of risk for the development of diabetes in the West of Scotland Coronary Prevention Study. Diabetes 2002;51:1596-1600.

[7] Frohlich M, Imhof A, Berg G, Hutchinson WL, Pepys MB, et al. Association between Creactive protein and features of the metabolic syndrome: a population-based study. Diabetes care 2000;23:1835-1839.

[8] Vu JD, Vu JB, Pio JR, Malik S, Franklin SS, et al. Impact of C-reactive protein on the likelihood of peripheral arterial disease in United States adults with the metabolic syndrome, diabetes mellitus, and preexisting cardiovascular disease. Am J Cardiol 2005;96:655-658.

[9] Voils SA, Cooper-DeHoff RM. Association between high sensitivity C-reactive protein and metabolic syndrome in subjects completing the National Health and Nutrition Examination Survey (NHANES) 2009-10. Diabetes Metab Syndr 2014;8:88-90.

[10] Jung CH, Lee WY, Kim SY, Shin HS, Kim HD, et al. The risk of metabolic syndrome according to the high-sensitivity $\mathrm{C}$-reactive protein in apparently healthy Koreans. Int J Cardiol 2008;129:266-271.

[11] Ridker PM, Wilson PW, Grundy SM. Should Creactive protein be added to metabolic syndrome and to assessment of global cardiovascular risk? Circulation 2004;109:2818-2825.

[12] Ye X, Yu Z, Li H, Franco OH, Liu Y, et al. Distributions of C-reactive protein and its association with metabolic syndrome in middleaged and older Chinese people. J Am Coll Cardiol 2007;49:1798-1805.

[13] Sigdel M, Kumar A, Gyawali P, Shrestha R, Tuladhar ET, et al. Association of high sensitivity C-reactive protein with the components of metabolic syndrome in diabetic and non-diabetic individuals. J Clin Diag Res 2014;8:Cc11-13.

[14] den Engelsen C, Koekkoek PS, Gorter KJ, van den Donk M, Salome PL, et al. High-sensitivity C-reactive protein to detect metabolic syndrome in a centrally obese population: a cross-sectional analysis. Cardiovasc Diabetol 2012;11:25.

[15] Mahajan A, Jaiswal A, Tabassum R, Podder A, Ghosh S, et al. Elevated levels of C-reactive protein as a risk factor for metabolic syndrome in Indians. Atherosclerosis 2012;220:275-281.

[16] Oda E. High-sensitivity C-reactive protein and white blood cell count equally predict development of the metabolic syndrome in a Japanese health screening population. Acta Diabetol 2013;50:633-638.

[17] Kawamoto R, Tabara Y, Kohara K, Miki T, Kusunoki $\mathrm{T}$, et al. Usefulness of combining serum uric acid and high-sensitivity C-reactive protein for risk stratification of patients with metabolic syndrome in community-dwelling women. Endocrine 2013;44:132-139.

[18] Alberti KG, Eckel RH, Grundy SM, Zimmet PZ, Cleeman JI, et al. Harmonizing the metabolic syndrome: a joint interim statement of the International Diabetes Federation Task Force on Epidemiology and Prevention; National Heart, Lung, and Blood Institute; American Heart Association; World Heart Federation; International Atherosclerosis Society; and International Association for the Study of Obesity. Circulation 2009;120:16401645.

[19] Consultation. WE. Appropriate body-mass index for Asian populations and its implications for policy and intervention strategies. Lancet 2004;363:157-163.

[20] Korea Centers for Disease Contrl and Prevention. The fourth Korea National Health and Nutrition Examination Survey. Cheongwon-gun: Korea Centers for Disease Contrl and Prevention, 2014; 121-122.

[21] Anand SS, Razak F, Yi Q, Davis B, Jacobs R, et al. C-reactive protein as a screening test for cardiovascular risk in a multiethnic population. Arterioscler Thromb Vasc Biol 2004;24:15091515.

[22] Khera A, McGuire DK, Murphy SA, Stanek HG, Das SR, et al. Race and gender differences in C-reactive protein levels. J Am Coll Cardiol 2005;46:464-469.

[23] Kelley-Hedgepeth A, Lloyd-Jones DM, Colvin A, Matthews KA, Johnston J, et al. Ethnic differences in C-reactive protein concentrations. Clin Chem 2008;54:1027-1037.

[24] Pearson TA, Mensah GA, Alexander RW, Anderson JL, Cannon RO, 3rd, et al. Markers of inflammation and cardiovascular disease: application to clinical and public health practice: A statement for healthcare professionals from the Centers for Disease Control and Prevention and the American Heart Association. Circulation 2003;107:499-511. 
[25] Sabatine MS, Morrow DA, Jablonski KA, Rice MM, Warnica JW, et al. Prognostic significance of the Centers for Disease Control/American Heart Association high-sensitivity C-reactive protein cut points for cardiovascular and other outcomes in patients with stable coronary artery disease. Circulation 2007;115:1528-1536.

[26] Pucci G, Alcidi R, Tap L, Battista F, MattaceRaso F, et al. Sex- and gender-related prevalence, cardiovascular risk and therapeutic approach in metabolic syndrome: A review of the literature. Pharmacol Res 2017;120:34-42.

[27] Aronson D, Bartha P, Zinder O, Kerner A, Markiewicz W, et al. Obesity is the major determinant of elevated C-reactive protein in subjects with the metabolic syndrome. Int $\mathbf{J}$ Obes Relat Metab Disord 2004;28:674-679.

[28] Zuliani G, Volpato S, Galvani M, Ble A, Bandinelli S, et al. Elevated C-reactive protein levels and metabolic syndrome in the elderly: The role of central obesity data from the InChianti study. Atherosclerosis 2009;203:626632.

[29] Florez H, Castillo-Florez S, Mendez A, Casanova-Romero P, Larreal-Urdaneta C, et al. $\mathrm{C}$-reactive protein is elevated in obese patients with the metabolic syndrome. Diabetes Res Clin Pract 2006; 71:92-100.

Citation: Hye-Sun Shin, Elevated Levels of High Sensitivity C-Reactive Protein are Associated with Metabolic Syndrome in a Representative Korean Sample.ARC Journal of Public Health and Community Medicine.2019; 4(1):37-45.DOI:dx.doi. org/10.20431/2456-0596.0401005.

Copyright: (C) 2019 Authors. This is an open-access article distributed under the terms of the Creative Commons Attribution License, which permits unrestricted use, distribution, and reproduction in any medium, provided the original author and source are credited. 